\title{
Secret Codes of Scale Distribution of Different Matter Stratums from Planck Scale to the Sun-Scale, Proof of Anthropic Principle and Different Stratum Sciences
}

\author{
Changyu Huang1*, Yong-Chang Huang2\# \\ ${ }^{1}$ Department of Physics and Astronomy, Purdue University, Lafayette, USA \\ ${ }^{2}$ Institute of Theoretical Physics, Beijing University of Technology, Beijing, China \\ Email: *cyhuang@purdue.edu, "ychuang@bjut.edu.cn
}

How to cite this paper: Huang, C. and Huang, Y.-C. (2017) Secret Codes of Scale Distribution of Different Matter Stratums from Planck Scale to the Sun-Scale, Proof of Anthropic Principle and Different Stratum Sciences. Journal of Modern Physics, 8, 1537-1546.

https://doi.org/10.4236/jmp.2017.89092

Received: May 2, 2017

Accepted: August 11, 2017

Published: August 14, 2017

Copyright $\odot 2017$ by authors and Scientific Research Publishing Inc. This work is licensed under the Creative Commons Attribution International License (CC BY 4.0).

http://creativecommons.org/licenses/by/4.0/

\begin{abstract}
This paper discovers the secret codes of the scale distribution from Planck scale to the Sun-scale by using Planck length derived from three fundamental physics constants, i.e., gravitation constant G, light speed c and Plank constant, and starting from the global consideration of treating the whole universe as a well unified entity in all scales. According to this symmetric scale distribution law of different scale regions: (a) we naturally give a possibility overcoming the difficulty of the desert effect between the grand unification scale and electroweak unification scale relevant to quarks and leptons, and it is really surprising to discover that the scales of quarks \& electrons, protons \& neutrons and atoms again all sequentially locate at the predicted points of the scale space; (b) we closely uncover the scale of the cells, which is the basic unit constructing the human bodies; (c) even the average height of human being is naturally deduced; (d) further, it is very surprising that the scales of the celestial bodies tightly related to us human beings, including the earth and the sun, also exactly fall at the predicted points in scale space in order. Therefore, all these scales with $10^{5 n} \mathrm{~cm}$ order $(\mathrm{n}=0,1,2, \ldots, 9)$ above just give a proof of very key anthropic principle for whole mankind (which just makes the anthropic principle be reduced as anthropic theorem), i.e., matter stratums (a) and (b) are inorganic and organic bases of constructing human being respectively; matter stratum (c) is just human being; matter stratums (d) are the living environments of human being. Namely, everything is for or relevant to the existence of human being. Consequently, the experimentally checked scale ladder of well-known matter levels just coincides with the scale ladder predicted by the deduced distribution law. From Planck scale to the Sun scale, people may
\end{abstract}


systematically build up the exact scientific theories corresponding ten matter stratums, may set up the different sciences among the different cross stratums, further, can systematically understand all the different sciences and their relations in the deepest way up to now.

\section{Keywords}

Astroparticle Physics, Particle Physics, Planck Scale, Basic Constant, Scale Secret Codes, Earth, The Sun, Anthropic Principle

\section{Introduction}

Through the development of Physics in hundreds of years, from the perspective of generally accepted physics theories, now we have gravitational theory, relativity and quantum mechanics, which give out the gravitational constant $G$, light speed $c$ and Plank constant $\hbar$, respectively. These three physical constants have been measured and checked by lots of strict physical experiments.

String theory can give the most hopeful complete description of the unified theory of everything in the universe, and provide a powerful toolbox along the way for solving problems in very different subjects, e.g., strongly and weakly coupled quantum field theories, cosmology and so on. Using the fundamental units of gravitational constant $G$, light velocity $c$ and Planck constant $\hbar$ in string theory, one can naturally obtain Planck length $l_{p}=\sqrt{G \hbar / c^{3}}$

$=1.61620 \times 10^{-33} \mathrm{~cm}$ [1]. This is just the length of fundamental superstring, the best candidate theory for now to describe quantum gravity, which, however, is conventionally taken as $10^{-34}$ in the past [2].

In modern science, due to the limit of human abilities, people divide the nature into different subjects to carry on independent research. Similarly, in modern physics, people divide it into different scales and separate it into unrelated small sections to investigate. Though many systems with multiple scales and correlations between different sections have been touched in the past, they were still limited and little work has been devoted to treating the universe of all the known scales as a whole integrated entity. This work is an attempt in this direction.

There are still various ways of dividing and defining different scale regions. They have their own reasons, which, though seemingly reasonable at first look, cannot survive after further investigations because they are short of profound scientific considerations and contain too strong artificial factors. Thus, the scale region division cannot be unified into one scheme and is, in fact, a mess. What's worse, these dividing methods are too coarse to be utilized to serve as a standard to undertake systematic and strict scientific research, or say, to carry on the separate research in every subfield of science, or at least in fields in the scale region from Planck scale to the Sun-star scale.

This paper wants to solve the above problems. The arrangement of this paper 
is: Section 2 is symmetries of different matter stratums with big scale gap from Planck scale to the planet-earth scale; Section 3 is investigations of stable matter stratums stabilized in different scales; Section 4 is proof of anthropic principle; the last Section is summary and conclusion.

\section{Symmetries of Different Matter Stratums with Big Scale Gap: From Planck Scale to the Planet-Earth Scale}

In order to research in a more systematic and strict way, it is necessary to do the investigations on the method of dividing different scale regions from the global perspective that treats stable scale matters from Planck scale to the Sun scale. Matters in these stratums can long keep stable, the other larger scales are greatly affected by the expansion of the universe. Thus we here don't consider the other scales above the Sun. In another word, we only consider the scales immune to the currently observable expansion effects of the universe, or scales where the expansion effect is negligible according to contemporary observation.

In order to find a starting point to do research on sciences in different scales under the consideration of treating the stable scale matters from Planck scale to the Sun scale as a whole entity, we take the more exact value $l_{p} \simeq 1.61620 \times 10^{-33} \mathrm{~cm}=a \times 10^{-33} \mathrm{~cm}$ as the value of Planck length, the unit length of the universe, instead of taking the conventional value $10^{-34} \mathrm{~cm}$. Furthermore, beginning from the $a \times 10^{-33} \mathrm{~cm}$ scale to larger scales, if we define the scale expanded by every $10^{20}$ times as a major scale, thus, we can naturally obtain Planck-scopic scale $a \times 10^{-33} \mathrm{~cm}$, Nucleon-scopic scale $a \times 10^{-13} \mathrm{~cm}$ and planet-earth-scopic scale $a \times 10^{7} \mathrm{~cm}$. Between the two adjacent major scales, more physics and/or the desert effect are naturally neglected, thus the divisions are too coarse.

Because there are always some inevitable errors for contemporary science in the measurements of physical quantities [3] [4] [5] and due to the influence of some small factors, there are commonly some perturbation effects to serve as some corrections for the dominative physics laws for the actual physical processes [5], so it is convenient to use order of magnitudes to describe and investigate stable scale matters in science.

\section{Symmetries of Different Matter Stratums with Stable Scale: From Planck Scale to the Star-Sun Scale}

Due to the complicatedness of the universe, people have to further focus on the phenomena at minor scales relative to the formerly defined major scales. Thus, beginning from the second major scale, i.e. Nucleon-scopic scale $a \times 10^{-13} \mathrm{~cm}$ above, for the times we define a series of minor scales by increasing the former scale $10^{5}$ times, i.e. $a \times 10^{-8} \mathrm{~cm}, a \times 10^{-3} \mathrm{~cm}, a \times 10^{2} \mathrm{~cm}$, etc.

As for the "scale space" between the first two major scales, Planck scale and Nucleon-scopic scale, we can only define one minor scale right below Nucleonscopic scale, i.e., the Quark-scopic scale $a \times 10^{-18} \mathrm{~cm}$, which is the smallest scale we can reach through current experiments (up to now, there are only some 
theoretical predictions about the scale space smaller than the Quark-scopic scale, but no experimental support). In this way, we can naturally obtain these scales and discuss their physics in each scopic scale in detail, including all the major and minor scales from the smallest to the larger, respectively, as follows:

For the most fundamental (i) Planck-scopic scale $a \times 10^{-33} \mathrm{~cm}$, it is the fundamental unit scale of superstring theory, which unifies the four fundamental interactions, and superstring theory is called the theory of everything, i.e. the theory is able to describe every kind of interactions in the universe. The matters participating different kinds of interactions are build up from the matters in this fundamental scale whose corresponding energy $E_{\text {Planck }}=1.22093 \times 10^{19} \mathrm{GeV}$ [2].

Moreover, up to now, all publically admitted physical laws have difficulties in keeping their effectiveness below Planck-scopic scale [2] [3] [4]. So, for now, there usually is not need to discuss the below Planck-scopic scale.

For (ii)-scale $a \times 10^{-28} \mathrm{~cm}$, its corresponding energy $E_{1}=\frac{E_{\text {Planck }}}{10^{5}}=1.22093 \times 10^{14} \mathrm{GeV}$, because the scale is also equal to $1 /$ energy in high energy physics [5], we surprisingly find that $E_{1}$ is just the grand unification energy scale, there are a lot of physical investigations and achievements in current references, e.g., see Ref. [5], so we don't repeat here. Specially, we here naturally deduce more exact magnitude value $E_{1}=1.22093 \times 10^{14} \mathrm{GeV}$ of the grand unification energy scale than $10^{15} \mathrm{GeV}$ of the current grand unification theory [5].

For (iii)-scale $a \times 10^{-23} \mathrm{~cm}$, it is just the preon scale, i.e., sub-quark scale.

Various models of preons have been proposed up to now [6] [7] [8] [9] [10], and we can find that the properties of quarks and leptons can be considered to be those of preons and preons' composites by analyzing the models above and the properties of quarks and leptons.

Similar to the introduction of quarks in constructing hadrons motivated by the fact that there exist too many basic hadrons and bosons, it is natural to introduce preons in constructing quarks and leptons from the viewpoint of quark and lepton classification because there exist too many basic quarks, leptons and mediating bosons [5].

Furthermore, because quarks and leptons have almost vanishing anomalous magnetic moments [5], which contradicts with the predictions of loosely binding state models, the forces of binding preons for forming the composited quarks and leptons must be effective at very short distance $l_{\text {Planck } 2}=a \times 10^{-23} \mathrm{~cm}$, it is just a scale of the binding forces, whose corresponding energy is $E_{2}=\frac{E_{\text {Planck }}}{\left(10^{5}\right)^{2}}=\frac{1.22093 \times 10^{19} \mathrm{GeV}}{\left(10^{5}\right)^{2}}=1.22093 \times 10^{9} \mathrm{GeV}$. This energy is the future accelerators' energy needed to do research on preon physics. This preon scale physics just gives a possibility overcoming the difficulty of the desert effect between the grand unification scale and the electroweak unification scale relevant to quarks and leptons, which was viewed as no new physics between the two scales above in the past. 
For (iv)-scale $a \times 10^{-18} \mathrm{~cm}$, it is right the quark scale since the upper bound of quark scale given by current experiments is $10^{-17} \mathrm{~cm}$ [5] and this result predicted by the current theory is consistent with experiments. Moreover, the experimentally proved particle physics standard model shows that quarks and electrons are in the same level of matter, and electrons do not participate in the strong interaction that needs color charge (and is therefore also called color interaction). Some theories (including Standard Model of Particle Physics) give out that electrons are just in a colorless singlet and are thus not affected by the exterior color interaction, namely, electrons are just like in a color saturate neutral state, and therefore the spatial scale of electrons can be larger. The logic here is somewhat similar to that electro-chargeless neutron has a larger spatial scale than electro-charged proton. This consideration makes the experimental result reasonable, in which the upper bound of electron scale $\left(10^{-16} \mathrm{~cm}\right)$ [5] is larger than that of quark scale $\left(10^{-17} \mathrm{~cm}\right)$.

For (v) Nucleon-scopic scale $a \times 10^{-13} \mathrm{~cm}$, it is just the scale of nucleon including protons and neutrons [1] [5], which is strictly consistent with physical experiments. Furthermore, since nuclei are made up of protons and neutrons through the residue interaction of strong (color) interaction, it can be naturally obtained that the scale of nuclei is at $a \times 10^{-12} \mathrm{~cm}$ which again well satisfies physical experiments [5].

For (vi)-scale $a \times 10^{-8} \mathrm{~cm}$ (1 $1 \AA$ ), it is right the scale of atoms [3] [5] [11], i.e. the Angstrom scale, and hence is also called Atom-scopic scale. This scale is consistent with physical experiments.

For (vii)-scale $a \times 10^{-3} \mathrm{~cm}$, it is the scale of big agglomerates consisting of clusters with their scales falling between tens of nanometer and hundreds of nanometer, or some other microscopic pellets. (The big agglomerates mentioned here include organic microscopic beings, such as the cells serving as basic building blocks of human bodies with the scale of $10-50$ micrometer, i.e. $(1 \sim 5) \times 10^{-3} \mathrm{~cm}$.) [12].

For (viii)-scale $a \times 10^{2} \mathrm{~cm}$, it is the scale of human beings, that is, the scale $10^{2} \mathrm{~cm}$ times the coefficient in front of the Planck length $a=1.616$ gives out the well-known average length of human beings [12]. This result agrees with experiments that everyone can see. It is necessary to especially point out that, according to statistics, if there is not any particular reason, the happening of one case should obey the principle of equal probability (or say opportunity). Thus, there are so many possible values of the human scale, but it just falls into the very tiny interval point of length space. Whether it is simply an amazing coincidence or there are some profound Physics really deserves to do some further research. Anyway, we can call this scale as Human-scopic scale.

For (ix)-scale $a \times 10^{7} \mathrm{~cm}$, the Earth-planet-scopic scale, the error is one order of magnitude smaller than the scale $10^{8} \mathrm{~cm}$ [13] of the earth. The increased one order of magnitude is originated from the bigger asteroid collision that produced the moon from the shivers of the colliding out during the formation process of 
the earth [13] [14]. There are many kinds of theories of the lunar origin [14] [15], but the popularly accepted theory is the colliding out theory of the lunar origin [14] [15], because there are a lot of observed facts of physical and astronomic experiments to support the colliding out theory of the lunar origin, e.g., Ref. [14] [15] [16]. It is very interesting to give out the origin of the moon here by the way.

For (x)-scale $a \times 10^{12} \mathrm{~cm}$, i.e., the star Sun-scopic scale [13], it is the scale of the star Sun obtained from astronomy observation, this scale is consistent with physical experiments.

For the scales above the star Sun-system-scopic scale, they are increasing with the universe's expansion, i.e., they are not stable scales, thus we don't discuss them here, but will study them in our following paper due to length limit of this paper.

\section{A Proof of Anthropic Principle}

Up to now, there are many kinds of anthropic principles and their investigations [17]-[22], we consider two dominate kinds of anthropic principles as follows:

Weak anthropic principle: The observed values of all physical and cosmological quantities are not equally probable but they take values restricted by the requirement that there exist sites where carbon-based life can evolve and by the requirements that the universe is old enough for it to have already done so [18].

Strong anthropic principle: The universe must have those properties which allow life to develop within it at some stage in its history [18].

According to this symmetric scale distribution law of different scale regions: (i $\&$ ii)-scales are the scales of superstring theory and grand unification theory of physics fundamental interactions, respectively; (iii-vi)-scales are the scales of inorganic matter bases of constructing human being; (vii)-scale is the scale of organic and inorganic matter bases of constructing human being; (viii)-scale is just the scale of human being; (ix \& $\mathrm{x}$ )-scales are the living matter bases of human being. Namely, everything seems designed for or at least is relevant to existence of human being.

Consequently, we can get everything of human being, including both the matter constructing human body and the matter circumstances of human being's life, simply from the following factors. Using gravitation constant $G$, light velocity $c$ and Planck constant $\hbar$, further utilizing natural number $\mathrm{n}=0,1, \ldots$, 9, and taking the other physics parameters, i.e., at least, 19 fundamental parameters of standard model of fundamental interactions: 1) three independent gauge coupling parameters $\mathrm{g}_{3}, \mathrm{~g}_{2}, \mathrm{~g}_{1}$ (because gauge groups of standard model are direct products of three different simple groups), 2) nine fermion masses; 3 ) four parameters of CKM matrix (three mix angles $\theta_{12}, \theta_{23}, \theta_{13}$ and one complex phase angle $\delta$ ); 4) two Higgs potential parameter (vacuum expection value $v$ and $\phi^{4}$ 's coefficient $\lambda$, or equivalent $\left.\left.m_{Z}, m_{H}\right) ; 5\right)$ one strong CP violation term parameter $\theta$ (or after renormalization $\theta^{\prime}$ ) [5]. 
Actually, the nineteen parameters and the natural numbers $n=0,1, \ldots, 9$ are not independent, i.e., they are relevant, and we can give the relations between the nineteen parameters and the natural numbers. Using the relations and the relevant parameters, we can create the universe when we can stipulate the relevant physics laws, initial conditions and boundary conditions. Also since Hawking has proved that the God is not needed, that is, after the relevant physics laws are stipulated and the initial conditions and boundary conditions are set, the universe can be automatically created, e.g., see Ref. [23].

Because we need human being, the matter bases constructing human being and their living environments, we need to, in wisdom, take the natural numbers and the above relevant physics parameters under the conditions that we can stipulate the relevant physics laws and given initial conditions and boundary conditions.

Because we have achieved the great achievements of all the current sciences after about 300 years from the capitalism industrial revolution, again after 300 years, 3000 years, 30,000 years or more, we certainly can reach the conditions above in terms of the development velocity of our sciences.

Specially, we now have partly got the feeling of being the God, namely, we can clone human bodies and various kinds of animals, furthermore, using gene technology, we can improve characters of all animals and plants, even including human being; furthermore, using feedback adjustable automation to replace Darwin's evolutionism, we can create biologic bodies from simple cell to many cells or from simple to complex automatically with some our needing adjustions, which make all our seeing everything be the result of Darwin's evolutionism; using large hadron colliders etc, we can even create the early vacuum of the universe, even create phase transition of the early vacuum, which may result in the appearance of the big bang of the early universe, when the accelerator's energy is high enough in future.

Therefore, we can create the universe, because we have the needs, i.e., our sun will become red giant star after 45 hundred million years, the great colliding between the milky way system and Andromeda galax will appear after about 34 hundred million years, our universe is expanding with acceleration [13] [24].

Our universe's fate will be big rip or big collapse etc. [24], which will not fit the living situation of human being. Therefore, at least, we have the motivation of investigating and experimentally creating the new universe to satisfy the surviving requirement of human being. If one civilization is advancing us about, at least, 138 hundred million years, whether they could also create our universe like we should do after 30,000 years or more some years. These problems are very interesting, which are worth to research carefully now and in the future.

\section{Summary and Conclusions}

Following the strict scientific methods of physics, this paper finds that all the known stable scale matters relative to human being in the universe appear ac- 
cording to a symmetric law, i.e., a new matter scale appears in every $10^{5}$ times in the scale space. In a word, we discover the secret codes of the scales of all the well-known stable scale matters relative to human being. It should be particularly emphasized that the reason why all the derived scales of already known matters relative to human being goes along well with all the observations is because our research is based on physical constants, obtained from strict physical experiments [5], and their derived scale-the Planck length.

Since this basis is also the foundational base of superstring theory (i.e., the theory of everything), the research results of this paper also show, from the global perspective of physics, that the basis of superstring theory is right, because utilizing the secret codes, people can predict new matter stratums.

Facing these coincidences, we have to emphasize:

1) It is very amazing that quarks, electrons, protons, neutrons, nuclei and atoms (of course they are the basic particles constructing our bodies) all appear at these interval points of some scope-scale regions. Why? It is too amazing for us to keep calm! Are quarks, electrons, protons, neutrons, nuclei and atoms also very special? And why should they be special?

2) The scale of the cells of human bodies just locates at the predicted point of the scale space. It is really surprising!

3) The scale of average height of human beings just falls at a predicted point of the scale space. It is really surprising!

4) What is more surprising is that the celestial bodies relating tightly to us, such as the earth and the sun (they are the exterior living environments of us) all locate at the predicted point of the scale space in order. Why? It is so interesting! Are the earth and the sun very special? And why should they be special?

5) Why do all the already known levels of stable scale matters relative to human being fall at these predicted points of the scale space without any exception? These phenomena are really interesting big accidents. What do these big "accidents" tell us? Or, is human being special in the universe? Or, is the universe special? All these above may be served as a proof for the existence of the anthropic principle of our observable universe.

6) The upcoming cosmological theory should explain these accidents, just as the e-fold numbers in cosmological inflation should be explained [24].

Are all the results and problems above simply an amazing coincidence or the consequence of some other reasons (maybe an intricate "design")? It is really a novel question! There must be some profound physics deserving further investigations. The solutions of these problems will undoubtedly be great progresses of our science. The solutions of these problems should depend on or at least be relevant to the symmetric distribution of all the studied scales in this paper. Particularly, the discoveries of the symmetrically distributed stable scales and their relations from Planck scale to the Sun scale provide a solid scientific platform for building up new branches of science and further development of old branches of science. From (i)-scale to (x)-scale people may exactly build up at least ten types 
of exact theories corresponding to every matter stratum, or, we, at least, may call the physics in every region as the corresponding scale physics, and for every region, people may build up many relevant exact scientific theories. So, the results of this paper are greatly useful and will be widely spread \& cited, because it will benefit \& promote developments of different branches of science, and because these results fit with current physical experiments and have great and practical physics meaning etc, they should eventually be written into relevant textbooks.

We specially need to stress that all the coincidences show the proof of anthropic principle, which are relevant to natural numbers $n=0,1,2, \ldots 9$, i.e., are wisdom choices, which just makes the essential anthropic principle for whole human being be reduced as anthropic theorem, but this is impossible in the past [24]. Furthermore, following this paper, there are lots of works to be done (due to length limit of this paper, these will be done in our or anyone's following works), e.g., we shall give the other new physics investigations relevant to $-\infty<$ $\mathrm{n}<\infty$, the relations among the fundamental parameters, the natural numbers etc, which will cancel their extra degrees of freedom, simplify relevant theories, and get the new physics landscape and the theories with the natural number marks (for the most direct cases, e.g., superstring theory with $n=0$, which means that this theory is best fundamental theory, i.e., the 0-level theory; the grand unification theory with $\mathrm{n}=1$, i.e., the 1-level theory, and so on). The results in this paper will help people to better understand the current branches of sciences and further systematically develop them in the deepest way up to now.

\section{Acknowledgements}

The work is supported by NSF through grants PHY-0805948, DOE through grant DE-FG02-91ER40681, National Natural Science Foundation of China (No. 11173028 and No.10875009) and the Project of Knowledge Innovation Program of Chinese Academy of Sciences (No. KJCX2. YW. W10).

\section{References}

[1] Cahn, R.N. (2009) The Experimental Foundations of Particle Physics. Cambridge University Press, Cambridge.

[2] Becker, K., Becker, M. and Schwarz, J.H. (2007) String Theory and M-Theory. Cambridge University Press, Cambridge.

[3] Woan, G. (2000) The Cambridge Handbook of Physics Formulas. Cambridge University Press, Cambridge.

[4] Dine, M. (2007) Supersymmetry and String Theory. Cambridge University Press, Cambridge.

[5] Particle Data Group (2012) Physical Review D, 86, Article ID: 010001. https://doi.org/10.1103/PhysRevD.86.010001

[6] Harari, H. and Seiberg, N. (1981) Nuclear Physics B, 100, 41.

[7] Hooft, G.T. (1980) Recent Development in Gauge Theories. Plenum Press, New York. https://doi.org/10.1007/978-1-4684-7571-5

[8] Harari, H. and Seiberg, N. (1982) Physics Letters B, 204, 141. 
[9] Pati, J.C., Cveti, M. and Sharatchandra, H.S. (1993) Physical Review Letters, 58, 851. Babu, K.S. and Pati, J.C. (1987) Physical Review D, 48, R1921. https://doi.org/10.1103/PhysRevLett.58.851

[10] Schmid, M.L. and Buchmann, A.J. (2009) Physical Review D, 80, Article ID: 095020. https://doi.org/10.1103/PhysRevD.80.095020

Sandin, F. and Hansson, J. (2007) Physical Review D, 76, Article ID: 125006. https://doi.org/10.1103/PhysRevD.76.125006

Das, C.R. and Laperashvili, L. (2006) Physical Review D, 74, Article ID: 035007. https://doi.org/10.1103/PhysRevD.74.035007

Bandos, I.A., de Azcárraga, J.A., Izquierdo, J.M., Picón, M. and Varela, O. (2004) Physical Review D, 69, Article ID: 105010. https://doi.org/10.1103/PhysRevD.69.105010 Parida, M.K. (1998) Physical Review D, 58, Article ID: 115006. https://doi.org/10.1103/PhysRevD.58.115006

[11] Nagashima, Y. (2010) Elementary Particle Physics. Wiley-VCH, Weinheim. Stock, R. (2009) Encyclopedia of Applied High Energy and Particle Physics. Wiley-VCH, Weinheim.

[12] McKinley, M. and O’Loughlin, V. (2009) Human Anatomy. McGraw-Hill, New York.

[13] Morison, L. (2008) Introduction to Astronomy and Cosmology. Wiley, Hoboken. Michael, Z. (1976) Astronomy-The Evolving Universe. Harper \& Row.

[14] Kaula, W.M. and Harris, A.W. (1975) Reviews of Geophysics, 13, 363. https://doi.org/10.1029/RG013i002p00363

[15] Schmitt, H.H. (2006) Moon's Origin and Evolution: Alternatives and Implications. In: Blondel, P. and Mason, JW, Eds., Solar System Update, Book Series: SpringerPraxis Books in Astronomy and Planetary Sciences, 111-134.

[16] Lee, D.C., Halliday, A.N., Snyder, G.A. and Taylor, L.A. (1997) Science, 278, 1098 1103. https://doi.org/10.1126/science.278.5340.1098

[17] Weinberg, S. (1987) Physical Review Letters, 59, 2607-2610. https://doi.org/10.1103/PhysRevLett.59.2607

[18] Barrow, J.D. (1997) Quarterly Journal of the Royal Astronomical Society, 24, 146153.

Oxford University Press (1986) Barrow \& Tipler's Definitions Are Quoted Verbatim at Genesis of Eden Diversity Encyclopedia. http://www.dhushara.com/book/quantcos/anth/anth.htm

[19] Carter, B. and McCrea, W.H. (1983) Philosophical Transactions of the Royal Society $A$, 310, 347-363. https://doi.org/10.1098/rsta.1983.0096

[20] Hogan, C. (2000) Reviews of Modern Physics, 72, 1149-1161. https://doi.org/10.1103/RevModPhys.72.1149

[21] Carr, B.J. and Rees, M.J. (1979) Nature, 278, 605-612. https://doi.org/10.1038/278605a0

[22] Livio, M. and Rees, M.J. (2003) Science, 309, 1022-1023. https://doi.org/10.1126/science.1111446

[23] Hawking, S. (1998) A Brief History of Time. University of Cambridge Press, Cambridge.

[24] Weinberg, S. (2008) Cosmology. Oxford University Press, Oxford. 
Submit or recommend next manuscript to SCIRP and we will provide best service for you:

Accepting pre-submission inquiries through Email, Facebook, LinkedIn, Twitter, etc. A wide selection of journals (inclusive of 9 subjects, more than 200 journals)

Providing 24-hour high-quality service

User-friendly online submission system

Fair and swift peer-review system

Efficient typesetting and proofreading procedure

Display of the result of downloads and visits, as well as the number of cited articles Maximum dissemination of your research work

Submit your manuscript at: http://papersubmission.scirp.org/

Or contact jmp@scirp.org 